\title{
Size Measurements of the Quasar X-Ray Continuum Emission Region by Analysis of Microlensing in Chandra Imagery
}

\author{
Chelsea L. MacLeod and Christopher W. Morgan \\ Physics Department, United States Naval Academy, 572c Holloway Rd, Annapolis, MD, USA \\ email: macleod@usna.edu, cmorgan@usna.edu
}

\begin{abstract}
Microlensing offers a unique way to constrain the physical extent of different emission regions in a lensed quasar, putting to test various accretion and continuum emission models. We perform a microlensing analysis using six Chandra observations (spanning six years) of the lensed quasar SDSS $0924+0219$ (redshift $z_{s}=1.52$ ), in which X-ray microlensing variability is detected with high confidence. The system exhibits pronounced microlensing variability in the X-rays compared to the optical, indicating a comparatively small extent of $r_{1 / 2}<3.8 \times 10^{14} \mathrm{~cm}$ ( $95 \%$ confidence) for the X-ray continuum emitting region, near the inner edge of the accretion disk.
\end{abstract}

Keywords. galaxies: active, quasars: general

\section{Introduction}

The structure of the quasar (Type I broad line AGN) continuum source remains an open question, especially at higher energies. Fortunately, microlensing offers a unique way to constrain the physical extent of different emission regions in a lensed quasar, putting to test various accretion and continuum emission models. Recent microlensing analyses in the X-rays revealed that the X-ray corona is compact relative to the optical emission (e.g., Morgan et al. 2012). Significant microlensing variability has been detected in optical observations of the quadruply lensed quasar SDSS 0924+0219 (Keeton et al. 2006, Morgan et al. 2006) and in recent Chandra monitoring data (Chen et al. 2012). Here, we model the X-ray microlensing in SDSS 0924+0219 and compare the size of the $\mathrm{X}$-ray emitting region to the accretion disk radius inferred from optical data in 2006.

\section{Data and Analysis}

For the analysis presented here, we use the full-band (0.4-8.0 keV) X-ray monitoring data for SDSS 0924+0219 provided by the ACIS imaging spectrometer (Garmire et al. 2003) on the Chandra X-Ray Observatory (Weisskopf et al. 2002) from six epochs between 2005 February 24 and 2010 October 5. These observations were a component of a larger Chandra Cycle 11 monitoring program, the details of which are published in Chen et al. (2012).

Our microlensing models are based on the relative tangential velocity between the source, lens galaxy stars, and observer, the convergence $(\kappa)$ and shear $(\gamma)$ near each of the lensed images as well as the stellar surface density fraction $\kappa_{*} / \kappa$, the relative size of the source, and the source plane projection of the Einstein radius $R_{E}$ of an average mass star $\langle M\rangle$ in the lens. We generate 40 magnification patterns over a sequence of $\kappa_{*} / \kappa$, using $8192^{2}$ pixels and an outer scale of $10 R_{E}$, yielding 400 total patterns and a pixel scale of $6.9 \times 10^{13}\left\langle M / M_{\odot}\right\rangle^{1 / 2} \mathrm{~cm}$. We model the uncorrelated variability in the 

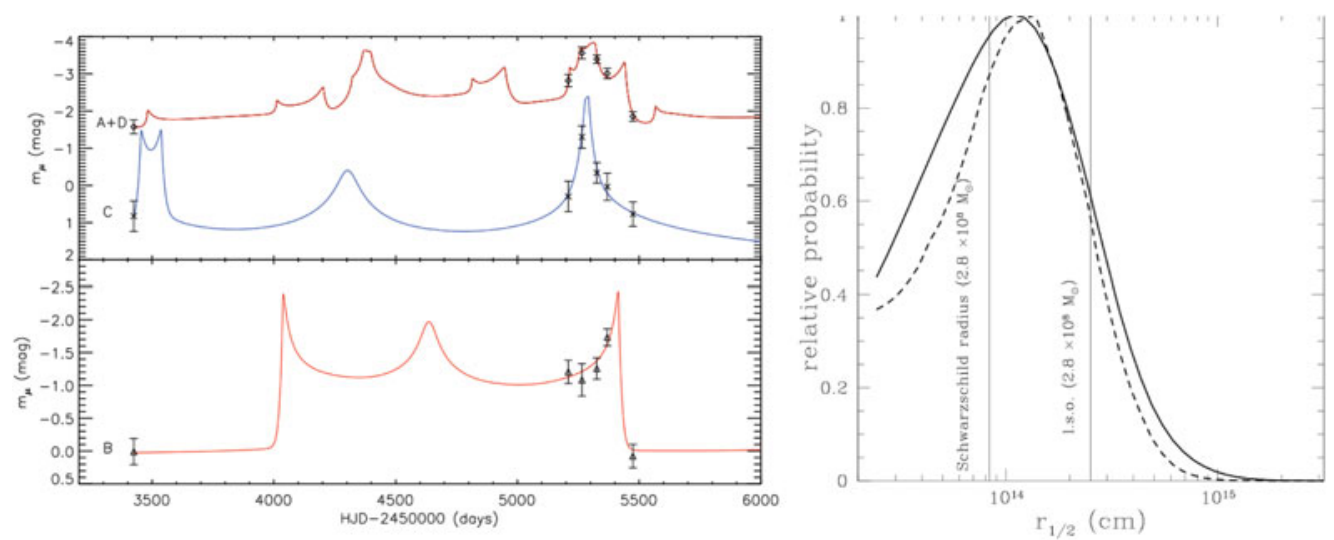

Figure 1. Left: Example X-ray microlensing model light curves (solid curves) for images A+D and C (top panels) and B (bottom panel). The symbols show the observed image flux minus our model for the intrinsic variability of the source. The model shown here uses a source size of $3 \times 10^{14}\left\langle M / M_{\odot}\right\rangle^{1 / 2} \mathrm{~cm}$ and $\kappa_{*} / \kappa=1$. Right: Probability distribution for the size of the X-ray-emitting region. The dashed line shows the result of imposing a prior on the mean microlens mass of $0.1<\left\langle M / M_{\odot}\right\rangle<1.0$. The vertical line shows the Schwarzschild radius $R_{B H}=2 G M_{B H} / c^{2}$ of a $2.8 \times 10^{8} M_{\odot}$ black hole (Morgan et al. 2006). The last stable orbit for a Schwarzschild black hole is at $3 R_{B H}$.

X-ray light curves as microlensing using the technique of Kochanek (2004) (we assume a negligible time delay between the images). Our Bayesian Monte Carlo analysis searches for trajectories across the patterns which yield model light curves that reproduce the observed data (e.g., see left panel of Fig. 1).

\section{Results and Implications}

After excluding fits with a $\chi^{2}$ per degree of freedom exceeding 3, our analysis of the full-band X-ray data yielded 3M solutions, $95 \%$ of which have half-light radii less than $3.8 \times 10^{14} \mathrm{~cm}$ (see Fig. 1, right panel). Meanwhile, the optical size was found to be significantly larger, in the range $6.4 \times 10^{14} \mathrm{~cm}<r_{1 / 2}<2.3 \times 10^{15} \mathrm{~cm}$ (Morgan et al. 2006), assuming an accretion disk inclination of $60^{\circ}$. Therefore, our result supports models involving a corona concentrated near the inner edge of the accretion disk, in agreement with recent results for other systems (e.g., Mosquera et al. 2013). The results from a joint microlensing analysis in the X-rays and optical (using recent ANDICAM and COSMOGRAIL $\dagger$ monitoring data) will be presented in an upcoming publication.

\section{References}

Chen, B., Dai, X., Kochanek, C. S., et al. 2012, ApJ, 755, 24

Garmire, G. P., et al. 2003, Proc. of the SPIE, 4851, 28

Keeton, C. R., Burles, S., Schechter, P. L., \& Wambsganss, J. 2006, ApJ, 639, 1

Kochanek, C. S. 2004, ApJ, 605, 58

Morgan, C. W., Kochanek, C. S., Morgan, N. D., \& Falco, E. E. 2006, ApJ, 647, 874

Morgan, C. W., Hainline, L. J., Chen, B., et al. 2012, ApJ, 756, 52

Mosquera, A. M., Kochanek, C. S., Chen, B., et al. 2013, ApJ, 769, 53

Weisskopf, M. C., Brinkman, B., Canizares, C., et al. 2002, PASP, 114, 1 